\title{
A Single Magnetic Nanocomposite Cilia Force Sensor
}

\author{
Ahmed Alfadhel ${ }^{1}$, Mohammed Asadullah Khan ${ }^{1}$, Susana Cardoso ${ }^{2,3}$, Jürgen Kosel ${ }^{1}$ \\ ${ }^{1}$ Computer, Electrical and Mathematical Sciences and Engineering Division (CEMSE) \\ King Abdullah University of Science and Technology (KAUST), Thuwal 23955, Saudi Arabia \\ ${ }^{2}$ INESC-Microsystems and Nanotechnologies (INESC-MN), Rua Alves Redol, 9, 1000-029 Lisbon, Portugal \\ ${ }^{3}$ Physics Department, Instituto Superior Técnico, Universidade de Lisboa, Lisbon, Portugal \\ *Email: ahmed.fadhel@kaust.edu.sa
}

\begin{abstract}
The advancements in fields like robotics and medicine continuously require improvements of sensor devices and more engagement of cooperative sensing technologies. For example, instruments such as tweezers with sensitive force sensory heads could provide the ability to sense a variety of physical quantities in real time, such as the amount and direction of the force applied or the texture of the gripped object. Force sensors with such abilities could be great solutions toward the development of smart surgical tools. In this work, a unique force sensor that can be integrated at the tips of robotic arms or surgical tools is reported. The force sensor consists of a single bioinspired, permanent magnetic and highly elastic nanocomposite cilia integrated on a magnetic field sensing element. The nanocomposite is prepared from permanent magnetic nanowires incorporated into the highly elastic polydimethylsiloxane. We demonstrate the potential of this concept by performing several experiments to show the performance of the force sensor. The developed sensor element has a $200 \mu \mathrm{m}$ in diameter single cilium with 1:5 aspect ratio and shows a detection range up to $1 \mathrm{mN}$ with a sensitivity of $1.6 \Omega / \mathrm{mN}$ and a resolution of $31 \mu \mathrm{N}$. The simple fabrication process of the sensor allows easy optimization of the sensor performance to meet the needs of different applications.
\end{abstract}

Keywords-Force Sensor, Smart Surgical Tools, Nanowires, Nanocomposite, Magnetic, Cilia

\section{INTRODUCTION}

The current progress in fields like robotics and biomedical engineering requires the development of smart force sensing technologies that combine miniaturization with low power consumption and high sensitivity. Force sensors with different sensing mechanisms have been introduced such as resistive, piezoelectric and capacitive sensing [1-6]. Many technical challenges still remain, including low sensitivity, limitations in detecting forces from all directions, in addition to the interference with environmental noise.

Cilia are micro-scale, hair-like structures that exist in nature in many forms. For example, the mechanosensorial receptors in spiders that are used to detect touch or vibration can provide outstanding interaction with the environment [7]. When the cilia are exposed to an external force from any direction, they bend and stimulate a neuron. This signal is then translated into a specific function.

Advances in material integration and nanofabrication technologies have enabled new directions of research and development for microsensor systems. Examples are nanocomposite materials, which combine the advantages of polymers-like flexibility, elasticity, chemical resistance or biocompatibility with the unique properties of the additive. Magnetic nanocomposites in which magnetic beads [8-10] or nanowires (NWs) [7, 11-13] are included in the polymer matrix enable remote control for heating, actuation or sensing. Fe NWs have a high magnetization in the absence of a magnetic field, due to the strong shape anisotropy, and they can be easily fabricated with a cost effective electrochemical process [14]. Incorporating these NWs in a polymer results in a permanent magnetic nanocomposite with distinct features and reinforced mechanical properties [7].

In this work, we report the fabrication and characterization of a robust and biomimetic force sensor that utilizes a permanent magnetic and highly elastic nanocomposite artificial cilium realized on a magnetic sensing element. The permanent magnetic properties of the nanocomposite are achieved by using $\mathrm{Fe}$ nanowires, which act as nano-scale permanent magnets and generate a magnetic stray field. The stray field, which is a function of the cilia's deflection, is detected by the giant magnetoresistive (GMR) sensor. The single-cilia force sensor has an active area of $100 \mu \mathrm{m} \times 100$ $\mu \mathrm{m}$ and consists of a $200 \mu \mathrm{m}$ in diameter cilium with 1:5 aspect ratio. Different cilia diameters and aspect ratios can be easily obtained with the facile fabrication process reported in this paper, enabling simple control of the sensors' performance and operating range and resolution. The unique concept combining cilia and magnetic sensing allows detecting shear and vertical forces in dry or wet environment, making it suitable for applications in medical tools or underwater robots. The DC operated sensor can detect static and dynamic forces as well as vibration and fluid flow.

A tactile sensor [7,12] and a flow sensor [11] have been reported previously using a similar concept but with an array of cilia integrated on a high frequency magnetic sensor to realize a single sensing element. Here we are moving toward miniaturization to a single cilium sensor, which provides a high spatial resolution. Moreover, the DC operation of the design reported in this paper simplifies the circuitry required for this type of sensors, and allows the realization of arrays with sensing pixels as small as $25 \mu^{2}$.

\section{METHODS}

\section{A. Sensor Concept}

The operating principle of the force sensor is based on detecting the change of the magnetic stray field, created by the permanent magnetic NWs, of the artificial cilia, when deflected by an external force (e.g. griping objects) (Fig. 1).

A DC operated GMR sensor is utilized to measure the change of the magnetic field upon deflection. At resting position, the stray field of the cilia provides a bias field for the GMR sensor, changing its initial resistance. In the presence of 
a force, the cilium bends in the force direction. This bending results in a change of the stray field of the cilium with an average magnetic field value affecting the GMR sensor, and hence changing its resistance.

(a)
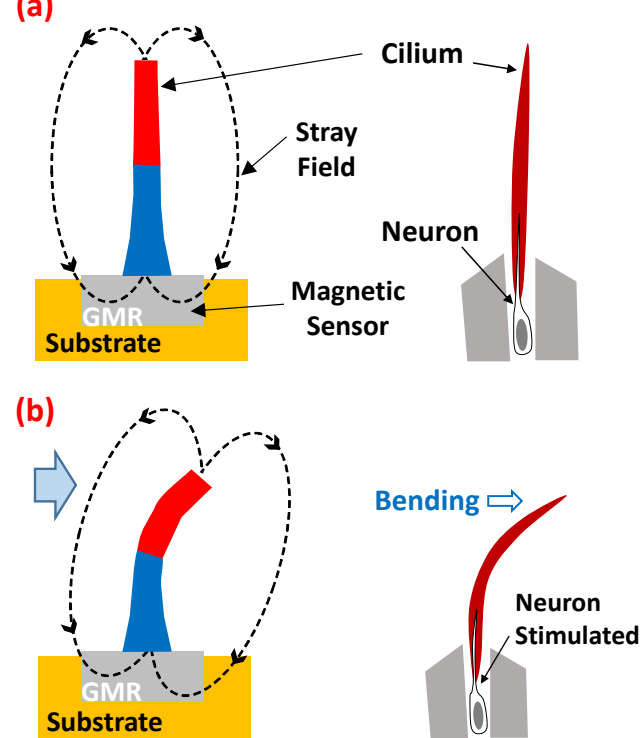

Fig. 1. Illustration of the operating principle of the magnetic cilia force sensor and comparing it to the microscale neuron-stimulated cilia we have in nature. (a) The sensor element is made of permanent magnetic nanocomposite cilia integrated on a magnetic sensor (magnetic North and South Pole are indicated by red and blue filling, respectively). (b) When the cilium gets deflected by a force such as fluid flow or contact with an object, the stray field at the giant magnetoresistive (GMR) sensor changes, and hence its resistance.

The deflection $(\delta)$ of the cilium due to a shear or vertical forces $F(x, y, z)$ is:

$$
\delta=\frac{64}{3} F_{x, y}(x, y, z) \frac{l^{3}}{\pi E D^{4}},
$$

where $l, E$, and $D$ are the length, Young's modulus and diameter of the cilium, respectively. Hence, the detection range and sensitivity can be easily controlled, by modifying the geometric or material properties of the nanocomposite cilium, i.e. the length, diameter or Young's modulus.

\section{B. Fabrication}

$6 \mu \mathrm{m}$ in length and $35 \mathrm{~nm}$ in diameter Fe NWs were fabricated by the electrodeposition into a nanoporous aluminum oxide membrane, prepared by a two-step anodization process using oxalic acid on an aluminum substrate (Fig. 2) [12]. Fe has been chosen for its high magnetization, biocompatibility and the possibility of efficient fabrication with this process. The NWs have been engineered to act as nanoscale permanent magnets, ensured by a single magnetic domain along the length of the NWs, This is achieved by keeping the diameter small, which also increases the intrinsic coercivity, making the NWs hard to demagnetize. The permanent magnetic NWs allow remote operation of the cilium sensor without an additional magnetic field to magnetize the nanowires, which simplifies miniaturization and system integration and minimizes the power consumption.

The nanocomposite was prepared by mixing NWs dispersed in sodium dodecyl sulfate surfactant with polydimethylsiloxane (PDMS) (Sylgard 184 Silicone Elastomer, Dow Corning Corporation) that was used as the polymeric matrix. The nanocomposite was cured at $90^{\circ} \mathrm{C}$ for 1 hour. The NWs to PDMS concentration is $16 \%$ volume ratio, providing a large magnetization of the nanocomposite without adversely affecting the polymerization process of the PDMS or the elasticity of the cilium. The NWs were aligned along the length of the cilium by applying a homogenous magnetic field of $0.8 \mathrm{kOe}$ during the curing process (Fig. 2).
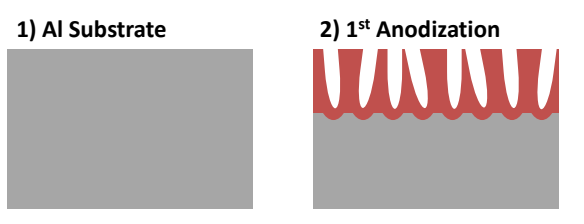

3) Alumina etch

4) $2^{\text {nd }}$ Anodization 5) Barrier reduction

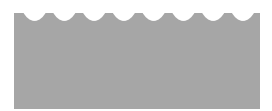

intoul in

6) NWs Electrodeposition

7) NWs Release

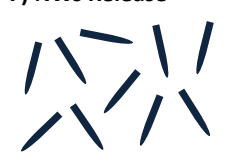

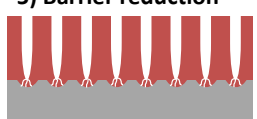

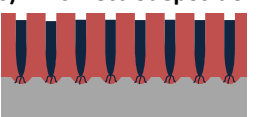

8) NWs mixing with polymer
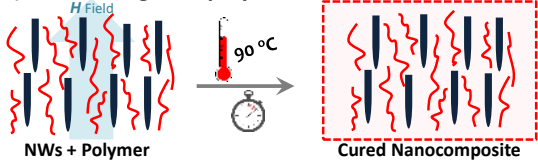

Fig. 2. Illustration of the electrochemical fabrication of Fe NWs and the preparation of the nanocomposite. An aluminum substrate is used (1) onto which two anodization steps are performed using oxalic acid to obtain uniform nanopores (2-4) of $\sim 35 \mathrm{~nm}$ in diameter, followed by a barrier reduction step for establishing an electrical contact with the bottom electrode (5). Fe is electrodeposited into the nanopores (6), and then the NWs are released by etching the alumina and dispersed in ethanol (7). The nanocomposite is fabricated by mixing the NWs with the polymer and then curing the composite. The NWs are aligned in a certain direction by applying an external homogenous magnetic field during the curing process (8).

A GMR magnetic field sensor array was fabricated by standard lithography, ion beam deposition and ion milling on a silicon substrate with the following structure (thickness in $\mathrm{nm}$, compositions in atomic \%): $\mathrm{Si} / \mathrm{Al}_{2} \mathrm{O}_{3} 60 / \mathrm{Ta} 1.0 / \mathrm{Ni}_{80} \mathrm{Fe}_{20}$ 2.8/ $\mathrm{Co}_{90} \mathrm{Fe}_{10}$ 2.5/ Cu 2.1/ $\mathrm{Co}_{90} \mathrm{Fe}_{10}$ 2.3/ $\mathrm{Mn}_{76} \mathrm{Ir}_{24}$ 7.0/ Ta 10.0 [15]. The metallic contacts were defined by lithography and liftoff of $300 \mathrm{~nm}$ thick $\mathrm{Al}_{98.5} \mathrm{Si}_{1.0} \mathrm{Cu}_{0.5}$ and $15 \mathrm{~nm}$ thick $\mathrm{Ti}_{10} \mathrm{~W}_{90}\left(\mathrm{~N}_{2}\right)$ layers. The GMR sensors array consists of 20 elements oriented for both $\mathrm{X}$ and $\mathrm{Y}$ sensing directions and is arranged within $100 \mu \mathrm{m} \times 100 \mu \mathrm{m}$ sensing area where each element is 5 $\mu \mathrm{m}$ long and $3 \mu \mathrm{m}$ wide. A thin PDMS layer of $\sim 5 \mu \mathrm{m}$ was then spun on top of the substrate to provide electrical isolation and, at the same time, enhance the adhesion of the cilium to the GMR sensor.

The nanocomposite cilium was fabricated using a $1 \mathrm{~mm}$ thick poly(methylmethcrylate) (PMMA) as a master mold into which a hole with $200 \mu \mathrm{m}$ in diameter is patterned with a $\mathrm{CO}_{2}$ laser cutter (Universal PLS6.75). The bottom diameter of the cilia is slightly wider than the top diameter, which is a result of patterning the mold with the laser beam. The nanocomposite was cast onto the surface of the GMR sensor and the master mold was mounted on top, thereby aligning the hole on top of the GMR sensor using a microscope. This structure was then placed in a desiccator for 30 minutes to remove any trapped air bubbles and assist in filling the hole. Next, the nanocomposite was cured at 90 degrees Celsius for one hour with an external magnetic field applied along the length of the cilium, forming the cilium with aligned NWs on top of the GMR sensor. After releasing the cured cilium, it was fully magnetized in one direction by applying a magnetic field of $10 \mathrm{kOe}$. The fabrication process is illustrated in Fig. 3. 
(I)
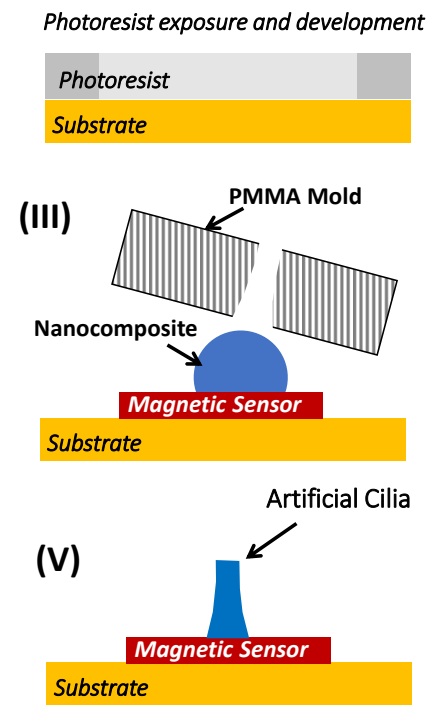

(II)

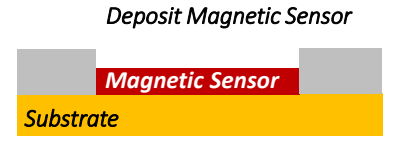

(IV)

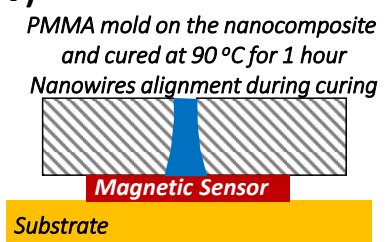

(VI)

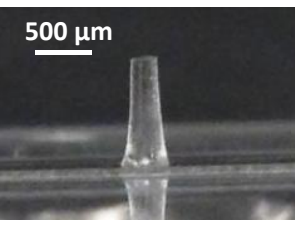

Fig. 3. Fabrication process of the magnetic cilium force sensor. (a,b) The magnetic sensor is fabricated using a standard lithography and deposition process. (b-e) The single cilium is fabricated using a PMMA mold. (f) Cilium with $200 \mu \mathrm{m}$ in diameter and 1:5 aspect ratio.

\section{Characterization}

In order to study the magnetic properties of the nanocomposite cilium, the magnetization curves along the length of the cilia and along the perpendicular direction were obtained using a vibrating sample magnetometer. As shown in Fig. 4, the cilium has a pronounced magnetic anisotropy with a remanence to saturation magnetization of $92 \%$ along the vertical direction and $35 \%$ along the horizontal direction with a high coercivity of $1.5 \mathrm{kOe}$. The anisotropic behavior confirms the alignment of the NWs along the cilium, and the high remanence value shows the permanent magnetic properties of the cilium. X-ray diffraction analysis reveals the NWs are polycrystalline Fe with a thin shell of magnetite (Fig. $5)$.

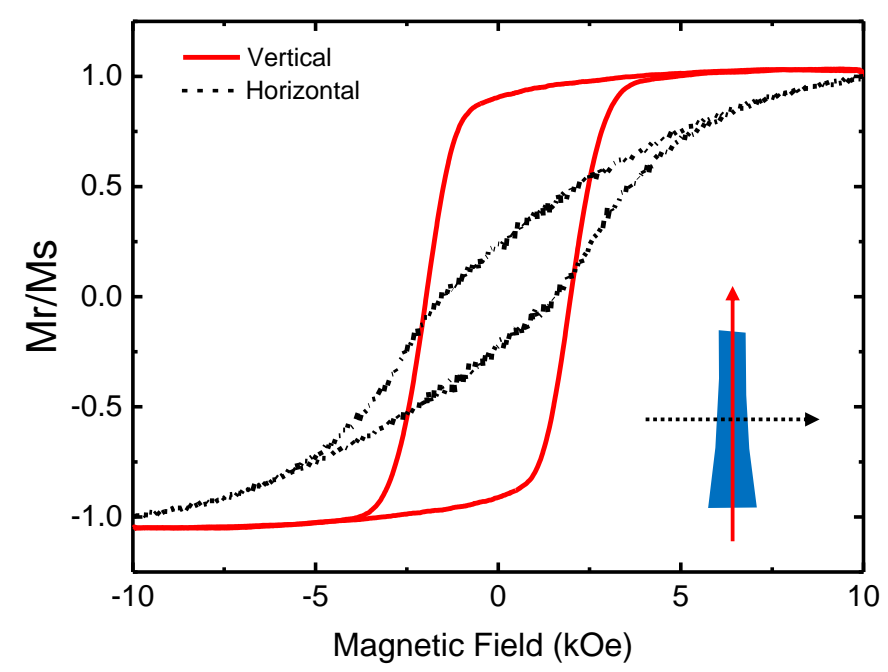

Fig. 4. Magnetization curves of magnetic nanocomposite cilia obtained with a vibrating sample magnetometer in the vertical and horizontal direction of cilia with vertically aligned NWs.

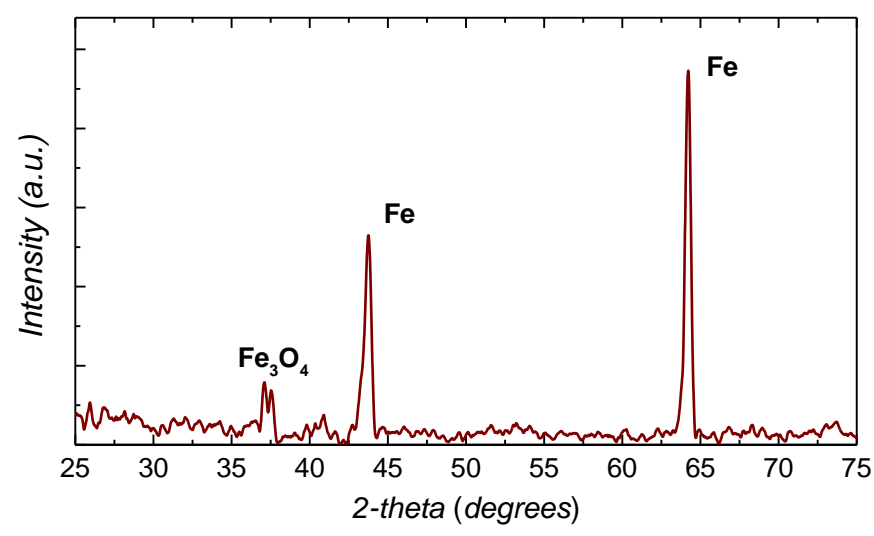

Fig. 5. X-ray diffraction results for a nanocomposite with Fe nanowires (Adapted from [12]).

In order to characterize the GMR sensor array, a Helmholtz coil was used to apply a magnetic field from 0 Oe to $18 \mathrm{Oe}$, while the resistance was obtained with a source meter (Keithley 2400) at a DC current of $0.1 \mathrm{~mA}$. The fields were applied in the $\mathrm{X}$ and $\mathrm{Y}$ directions with respect to the substrate (Fig. 6). The response of the GMR sensor array shows a sensitivity of $2.5 \Omega / \mathrm{Oe}$ in the $\mathrm{X}$ direction and 1.9 $\Omega / \mathrm{Oe}$ in the $\mathrm{Y}$ direction. This isotropic magnetic sensitivity provides the ability to detect the magnetic signal that is being measured in all directions.

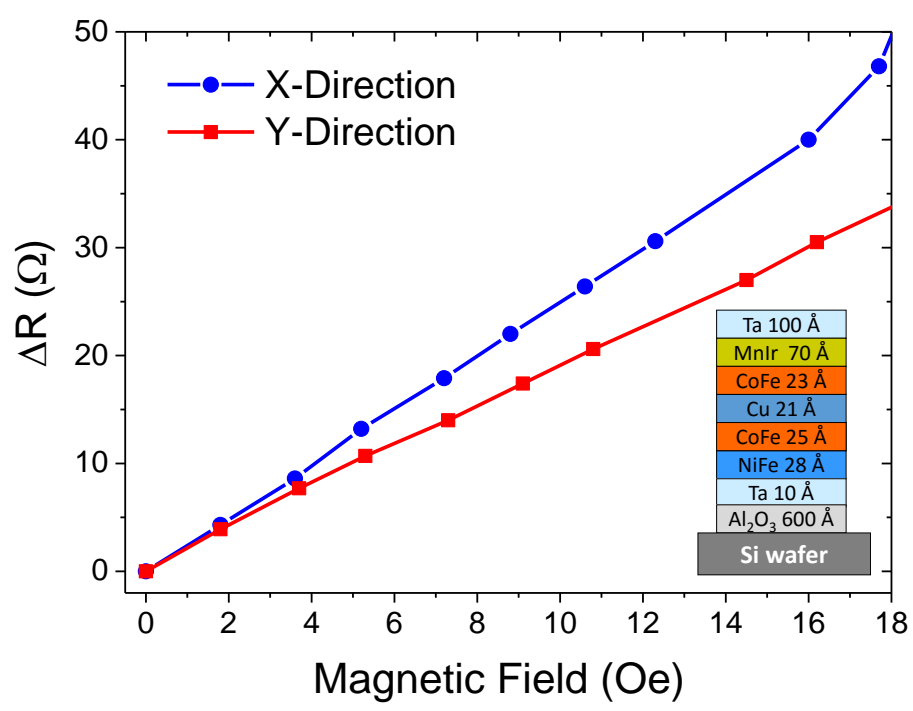

Fig. 6. Resistance response of the GMR sensor array with an external magnetic field applied to the $\mathrm{X}$ and $\mathrm{Y}$ directions. Inset: The fabricated GMR sensor stack.

\section{RESUlTS}

The force sensor was tested by applying loads using a computer controlled stepping motor INSTRON extensometer (tensile/compressive force testing system) with a force sensing feedback. The system can precisely determine the exerted force and the vertical displacement. The resolution values depend on the sample dimensions and the utilized load cell. By using a $10 \mathrm{~N}$ load cell and a cilium that is $1 \mathrm{~mm}$ long and $200 \mu \mathrm{m}$ in diameter, a compressive force resolution of $31 \mu \mathrm{N}$ and vertical displacement of $56 \mu \mathrm{m}$ can be measured. A DC current of $0.1 \mathrm{~mA}$ was applied to the sensor through an SMA port and the resistance was measured directly using a source 
meter (Keithley 2400) connected via a shielded BNC-SMA cable adapted with banana plugs to the source meter. No significant variations were observed in the signal when moving the sensor around with fluctuation less than $0.05 \Omega$. As shown in Fig. 7, the single cilium sensor can detect forces between $0-1 \mathrm{mN}$ with a sensitivity of $1.6 \Omega / \mathrm{mN}$ and a high resolution of $31 \mu \mathrm{N}$, which was calculated by considering the noise in the system $( \pm 0.05 \Omega)$. A linear range from $0 \mathrm{mN}$ to $0.85 \mathrm{mN}$ is observed with a maximum resistance change of 1.4 $\Omega$ before the response saturates representing the full deflection of the cilium.

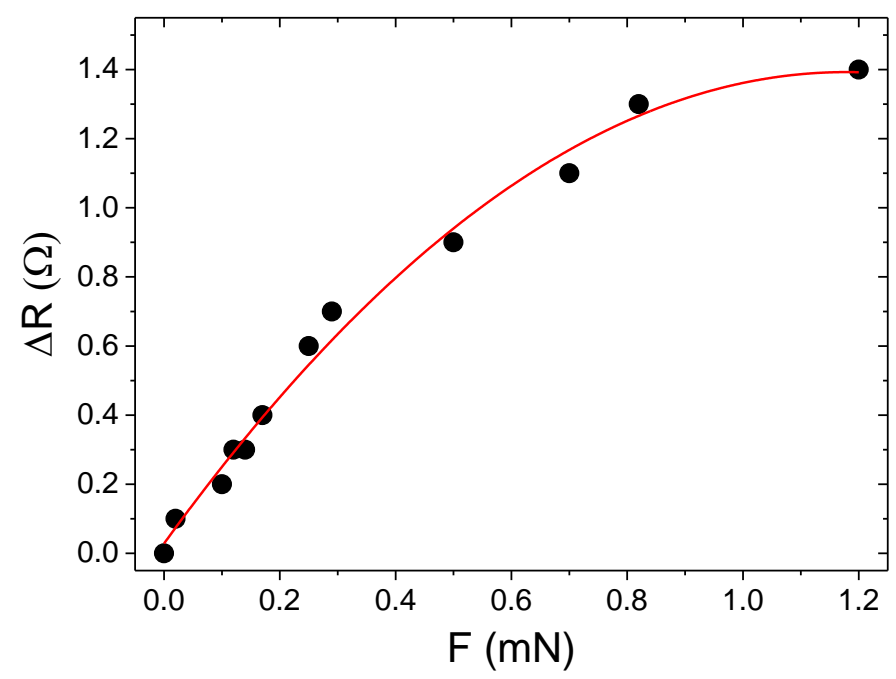

Fig. 7. Experimental results of the cilium force sensor. Resistance change upon application of vertical forces to the cilium.

\section{CONCLUSION}

A NWs-based permanent magnetic and highly elastic nanocomposite has been used to realize a single cilium on top of a GMR sensor for force sensing applications. This unique cilium sensor concept could be useful in, e.g., flow measurements or tactile sensing for robotics, medical or biological applications. The permanent magnetic behavior of the NWs enables detecting the deflection of the cilia with the GMR sensor. This greatly simplifies miniaturization and minimizes the power consumption. The properties of the nanocomposite can be tailored in a wide range by the choice of the materials and the dimensions of the cilium as well as by the concentration of the NWs. Therefore, the developed sensor has an easily modifiable sensing range and resolution. The results of the force sensor show a detection range up to $1 \mathrm{mN}$ with a sensitivity of $1.6 \Omega / \mathrm{mN}$ and a resolution of $31 \mu \mathrm{N}$. The cilium buckles easily in the first region and the sensitivity reduces as it gets harder to fully deflect the cilium until reaching the maximum deflection, resulting in signal saturation.

\section{ACKNOWLEDGEMENTS}

Research reported in this publication was supported by the King Abdullah University of Science and Technology (KAUST).

\section{REFERENCES}

[1] S. Mohsin, I. Tiwana, and N. Lovell, "A review of tactile sensing technologies with applications in biomedical engineering," Sens. Actuators A, vol. 179, pp. 17, 2012.

[2] K. Takei, et al., "Nanowire active-matrix circuitry for low-voltage macroscale artificial skin," Nature Materials, vol. 9, pp. 821, 2010.

[3] C. Pang, et al., "A flexible and highly sensitive strain-gauge sensor using reversible interlocking of nanofibres," Nature Materials, vol. 11, pp. 795, 2012.

[4] B. Tee, et al., "An electrically and mechanically self-healing composite with pressure- and flexion-sensitive properties for electronic skin applications," Nature Nanotechnology, vol. 7, pp. 825, 2012.

[5] M. Segev-Bar, A. Landman, M. Nir-Shapira, G. Shuster, and H. Haick, "Tunable Touch Sensor and Combined Sensing Platform: Toward Nanoparticle-based Electronic Skin,"ACS Applied Mater. \& Inter., vol. 5, pp. 5531, 2013.

[6] H. Kim, S. Lee, and K. Yun, "Capacitive tactile sensor array for touch screen application," Sens. and Actuators A, vol. 165, pp. 2, 2011.

[7] A. Alfadhel, and J. Kosel, "Magnetic Nanocomposite Cilia Tactile Sensor," Adv. Mater., vol. 27, pp. 7888-7892, 2015.

[8] Y. Yi, A. Zaher, O. Yassine, J. Kosel, and I.G. Foulds, "A remotely operated drug delivery system with an electrolytic pump and a thermoresponsive valve," Biomicrofluidics, vol. 9, pp. 052608, 2015.

[9] B. Zhou, et al., " Design and fabrication of magnetically functionalized flexible micropillar arrays for rapid and reversible microfluidic mixing," Lab Chip, 2015.

[10] A. Zaher, S. Li, O. Yassine, N. Khashab, N. Pirmoradi, L. Lin, and J. Kosel: "Osmotically driven drug delivery through remote-controlled magnetic nanocomposite membranes". Biomicrofluidics, vol. 9, pp. 5, 2015.

[11] A. Alfadhel, B. Li, A. Zaher, O. Yassine, and J. Kosel, "Magnetic nanocomposite for biomimetic flow sensing," Lab Chip, vol. 14, pp. 4362-4369, 2014.

[12] A. Alfadhel, and J. Kosel, "Magnetic Micropillar Sensors for Force Sensing," 2015 IEEE Sensors Applications Symposium (SAS), Zadar, Croatia, 12-15 April, 2015.

[13] M. Alnassar, A. Alfadhel, Yu. P. Ivanov, and J. Kosel "Magnetoelectric Polymer Nanocomposite for Flexible Electronics," J. Appl. Phys. Vol. 117, pp. 17D711, 2015.

[14] S. Thongmeea, H.L. Panga, J. Dinga, and J.Y. Lin "Fabrication and magnetic properties of metallic nanowires via AAO templates," $J$. of Mag. and Mag. Mat., vol. 321, pp. 2712-2716, 2009.

[15] P. Freitas, R. Ferreira, S. Cardoso, and F. Cardoso, "Magnetoresistive sensors", Journal of Physics: Condens. Matter, vol. 19, pp. 165221, 2007. 